\title{
USO DO MÉTODO ELECTRE III PARA ESCOLHA DE UMA ESTRATÉGIA DE NAVEGAÇÃO DE BARCOS DE APOIO ÀS UNIDADES MARÍTIMAS
}

\author{
Pablo Tentempo Steiner \\ Programa de Engenharia de Produção/Coppe - UFRJ -Rio de Janeiro -RJ \\ Av. Horácio Macedo, 2030 - Cidade Universitária \\ pablo.stb@gmail.com \\ Ayala Liberato Braga \\ Programa de Engenharia de Produção/Coppe - UFRJ -Rio de Janeiro -RJ \\ Av. Horácio Macedo, 2030 - Cidade Universitária \\ ayala.braga@gmail.com \\ Alexandre Portela de Abreu \\ Programa de Engenharia de Produção/Coppe - UFRJ -Rio de Janeiro -RJ \\ Av. Horácio Macedo, 2030 - Cidade Universitária \\ Alexandre30portela@gmail.com \\ Igor Girão Peres Vianna \\ Programa de Engenharia de Produção/Coppe - UFRJ -Rio de Janeiro -RJ \\ Av. Horácio Macedo, 2030 - Cidade Universitária \\ igor_gpv@poli.ufrj.br \\ Tharcisio Cotta Fontainha \\ Programa de Engenharia de Produção/Coppe - UFRJ -Rio de Janeiro -RJ \\ Av. Horácio Macedo, 2030 - Cidade Universitária \\ tharcisio.fontainha@gmail.com
}

\section{RESUMO}

Com o crescimento do setor de petróleo no mundo e no Brasil as atividades em ambiente offshore mostram que existe uma gama de dificuldades nas operações logísticas neste meio produtivo. Dada a grande quantidade de variáveis e de dados utilizados nas atividades que integram toda a cadeia operacional na área offshore podem ser utilizados modelos de pesquisa operacional a fim de encontrar soluções otimizadas. Para estes casos se faz necessária o uso de uma análise multicritério, podendo então ser considerados fatores econômicos, fatores sustentáveis dentre outros. Assim, o trabalho busca contribuir no ordenamento de três estratégias de seleção da velocidade de navegação da frota de PSVs (Plataform Supply Vessels) que são barcos que suprem as demandas das Unidades Marítimas (UMs), baseando-se em critérios econômicos, ambientais e de bem-estar da tripulação, analisados por um modelo multicritério. Para alcançar este objetivo, foi utilizada uma ferramenta de apoio à decisão denominada APOLO, que combina um simulador de eventos discretos com uma heurística de alocação dos PSVs às cargas e às unidades marítimas. Foi selecionado o modelo ELECTRE III para a realização da análise multicritério e depois foram definidos os indicadores relevantes para o problema. Como resultado obteve-se a terceira estratégia como vencedora, apresentando os índices de concordância e as relações de preferência entre as estratégias de navegação. Deste modo, as informações obtidas pelo modelo podem contribuir para a tomada de decisão e que realçam a viabilidade do emprego do método para análise de tal problema. O método multicritério se mostrou relevante e aplicável a processos de tomada de decisão que envolvam variáveis de caráter subjetivo e 
quantitativo.

Palavra-chave: ELECTRE III; Logística; Multicritério; Unidades Marítimas; Simulação.

\begin{abstract}
With the growth of the oil sector in the world and Brazil, activities in the offshore environment show that there is a range of difficulties in logistics operations in this production environment. Given a large number of variables and data used in the activities that integrate the entire operational chain in the offshore area, operational research models can be used to find optimized solutions. For these cases, it is necessary to use a multicriteria analysis, which can then be considered economic factors, sustainable factors among others. Thus, this work seeks to contribute to the scheduling of three strategies of the selection of the navigation speed of the fleet of PSVs (Platform Supply Vessels), boats that supply the demands of the Maritime Units, based on economic, environmental and crew welfare, analyzed by a multicriteria model. To achieve this goal, a decision support tool called APOLO was used, which combines a discrete event simulator with a PSVs heuristic for cargo and marine units allocation. The ELECTRE III model was selected for the multicriteria analysis, then the relevant indicators for the problem were defined. As a result, we obtained the third strategy as the winner, presenting the agreement indices and the preference relations between the navigation strategies. Thus, the results obtained by the model can contribute to take decisions. The multicriteria method was relevant and applicable to decision-making processes that involve subjective and quantitative variables.
\end{abstract}

Keywords: ELECTRE III; Logistic; Multicriteria; Maritime Units; Simulation.

\title{
Como Citar:
}

STEINER, P.T.; BRAGA, A.L.; DE ABREU, A.P., VIANNA, I.G.P.; FONTAINHA, T.C. Uso do método electre iii para escolha de uma estratégia de navegação de barcos de apoio às unidades marítimas. In: SIMPÓSIO DE PESQUISA OPERACIONAL E LOGÍSTICA DA MARINHA, 19., 2019, Rio de Janeiro, RJ. Anais [...]. Rio de Janeiro: Centro de Análises de Sistemas Navais, 2019.

\section{INTRODUÇÃO}

O setor de petróleo vem crescendo continuamente no mundo e no Brasil, com um crescimento de 3,2\% na comparação anual. Porém, com o passar dos anos as reservas de mais fácil exploração foram se esgotando. No atual cenário brasileiro, a grande maioria da produção atual, 94\% do total, e das futuras reservas se encontra em ambiente offshore, sendo o pré-sal responsável por $40,6 \%$ da produção nacional, que é um dos mais complicados de serem explorados devido as grandes profundidades a serem perfuradas e aos efeitos do mar na corrosão de equipamentos e tubos e na instabilidade ocasionada pelas ondas (ANP,2017). Além de todos os complicadores mencionados, ainda há uma gama de dificuldades na logística de operação em grande escala nesse ambiente.

Dessa forma, as atividades de Exploração e Produção de Óleo e Gás (E\&P) no setor offshore têm imposto grandes desafios relacionados à área de planejamento e operação de 
recursos e infraestrutura logísticos, que são áreas fundamentais para a sua continuidade operacional, podendo causar, por exemplo, paradas de produção e atraso no cronograma de perfuração de determinado poço (ARES, 2013). Nesse contexto, a logística de apoio offshore, ou apenas logística offshore pode ser dividida em três grandes tipos de operações: logística de cargas, logística de passageiros e serviços.

Para as operações de transporte e abastecimento, que se encontram dentro da logística de cargas, realizadas para as unidades marítimas (UMs), são utilizadas embarcações supridoras, chamadas de barcos de apoio ou de PSVs (Plataform Supply Vessels), sendo um dos elementos mais custosos de cadeia logística offshore.

O aumento das operações offshore se não for planejado e bem coordenado, pode gerar inúmeros problemas econômicos e também sustentáveis como: atrasos nas entregas dos produtos; maior consumo de combustível por parte da frota e consequentemente maior emissão de gases poluentes; maiores períodos embarcados da tripulação; dentre outros. Muitos desses problemas são impactados diretamente pela velocidade de navegação do PSV.

Dada a importância do setor de E\&P, a grande quantidade de variáveis e de dados utilizados e as atividades que integram toda a cadeia operacional na área offshore, o planejamento pode se tornar extremamente complexo, sendo necessária a utilização de modelos de pesquisa operacional a fim de encontrar soluções otimizadas para o empreendimento. Esta abordagem se faz ainda mais necessária quando se visa avaliar indicadores, que, a priori, não poderiam ser analisados em conjunto. Para estes casos se faz necessário o uso de uma análise multicritério de avaliação, podendo então considerar fatores econômicos e fatores sustentáveis ao mesmo tempo.

A principal contribuição deste trabalho é o ordenamento de diferentes estratégias de seleção da velocidade de navegação da frota, baseando-se em critérios econômicos, ambientais e de bem-estar, analisados por um modelo multicritério.

Para alcançar este objetivo, cabe identificar primeiramente o método de apoio à decisão mais adequando. Watrobski et al. (2019) discute a existência de diversos métodos, tais como AHP, ELECTRE I, ELECTRE II, ELECTRE III, PROMETHEE I, PROMETHEE II, etc, e que sua utilização é indicada de acordo com características do problema, de precisão/incerteza das variáveis envolvidas, etc. A partir das características do problema, verifica-se como adequada a utilização uma ferramenta de apoio à decisão, denominada APOLO e apresentada em Vianna (2019), que combina um simulador de eventos discretos com uma heurística de alocação dos PSVs às cargas e às unidades marítimas. No que tange o método de apoio à decisão, é selecionado o modelo ELECTRE III para a realização da análise multicritério, depois são definidos os indicadores relevantes para o problema, um para cada um dos critérios propostos. Então são definidas três estratégias de determinação da velocidade de navegação do $P S V$.

Na sequência deste artigo é apresentada uma revisão sobre a logística offshore e métodos multicritérios, seguida pela definição do problema estudado. Na seção 4 são apresentados os experimentos computacionais conduzidos. Encerra o artigo as conclusões e sugestões de trabalhos futuros.

\section{REVISÃO BIBLIOGRÁFICA}

Aguiar (2013) apresenta as atividades de apoio offshore como as atividades de transporte e armazenagem de cargas realizadas por embarcações de apoio para realizar serviços de E\&P nas plataformas offshore.

Segundo Almeida (2009), as atividades de E\&P offshore de petróleo e gás demandam uma logística muito complexa no suprimento de alimentação, água, cargas em 
geral (peças de reposição, equipamentos, tubos, etc.), óleo diesel e outros. O suporte marítimo destaca-se como um elo importante na cadeia logística que garante o nível de serviço desejado nas operações de suprimento Di Luccio e Dores (2016), sendo vital o planejamento eficiente da logística para suprimento de material para a exploração e produção Arpini e Rosa (2015), no qual atrasos ou falhas nas entregas podem acarretar enormes prejuízos financeiros e ambientais (BRAGA, 2017).

Assim, essa logística de apoio vem se tornando uma nova área de conhecimento no segmento de Óleo e Gás (O\&G), onde cada vez mais as empresas de E\&P de petróleo demandam elevados níveis de serviço nas atividades de suporte à operação de exploração e produção (COSTA, 2015).

Para atender essa demanda, Almeida (2002) demonstra que métodos multicritérios de apoio à decisão se adequam às situações em que nem todos os dados de um determinado problema prático podem ser facilmente quantificados, por medição ou estimativa e, onde tais situações frequentes nos processos decisórios das empresas e são geralmente complexos. A aplicação de métodos Múltiplos Critérios é pertinente em situações em que se necessita da análise de múltiplos (dois ou mais) critérios, ou múltiplos atributos (MARTINS e COELHO, 2012).

Visando entregar esses elevados níveis de serviços se faz necessário também a utilização de simuladores de eventos para minimizar os erros. Dessa forma, Silva et al. (2015) compararam várias políticas de transporte marítimo, empregando a simulação de eventos discretos para estimar o dimensionamento da frota e a infraestrutura necessárias para suprir a demanda de carga.

De acordo com Pinto et al. (2015), grandes distâncias no deslocamento e o alto custo envolvido nessas operações demonstram a importância de se trabalhar com a otimização do dimensionamento de barcos de apoio marítimo e do roteamento desta frota, melhorando o nível de serviço. Assim, eles propõem um modelo matemático baseado no Problema de Roteamento de Veículos Periódicos (PRVP) para elaboração de viagens diárias de barcos às plataformas offshore, bem como a definição de tamanho e composição da frota para atender a demanda.

Silva et al. (2015) comparam várias políticas de transporte marítimo, empregando a simulação de eventos discretos para estimar o dimensionamento da frota e a infraestrutura necessárias para suprir a demanda de carga.

Moreira (2017) mostra como é possível unir frotas de navios e carga geral, sem deteriorar o nível de serviço. Vários cenários são avaliados utilizando-se a simulação de eventos discretos. Como resultado, uma notável redução dos custos é observada, graças ao significante corte na frota original de PSVs estimada pelo trabalho.

Em Norlund et al. (2015) é analisado como a otimização da velocidade das embarcações pode reduzir o consumo de combustível e emissões de CO2. Em um trabalho posterior, Norlund e Gribkovskaia (2017) estendem essa análise em como a programação do porto pode ser construída visando diminuir o consumo de combustível baseado em diferentes técnicas de otimização de velocidade das embarcações. Em ambos os trabalhos, a condições meteorológicas é levada em conta na simulação.

Eskandari e Mahmoodi (2016) também utilizam um simulador de eventos discretos que leva em conta condições ambientais e uma estratégia de otimização baseada em simulação para achar a composição da frota que minimize o custo total mantendo um nível de serviço mínimo.

Norstad et al. (2017) apresentam um estudo feito para o mar do ártico onde são simulados vários formatos dos navios e composições de frota respeitando alguns requerimentos de segurança, preparações emergenciais e indicadores ambientais levando em conta o impacto do clima nos tempos de navegação e no consumo de combustíveis. 
Kisialiou et al. (2018) visam minimizar o número de embarcações e o gasto com combustíveis ao mesmo tempo que mantém um nível mínimo de serviço.

Vianna (2019) analisa diferentes estratégias, entre heurísticas gulosas e modelo de otimização, de alocação de PSVs às cargas e às unidades marítimas com o auxílio de simulação de eventos discretos baseados nas operações da Petrobras no litoral do Rio de Janeiro e de Espírito Santo.

\section{DEFINIÇÃO DO PROBLEMA}

Para uma melhor definição do problema, ele é dividido em três partes principais:

a) Definição conceitual: é apresentada uma definição geral do problema e das etapas propostas para a solução do mesmo; trabalho;

b) Modelagem da operação offshore: explicita as operações relevantes para este

c) Modelo multicritério: É apresentado o modelo multicritério a ser utilizado.

\subsection{DEFINIÇÃO CONCEITUAL}

As UMs são responsáveis pelas operações de E\&P da indústria de petróleo e necessitam de uma gama grande e variada de cargas, denominadas de carga geral, que apresentam datas de entrega definidas, elas também produzem cargas que precisam ser retornadas para terra, as cargas de backload. Além disso, as UMs apresentam demandas por diesel para combustível e água para suas operações internas, sendo possível que algumas delas sejam capazes de produzir uma parte da água utilizada para consumo.

As demandas e necessidades das UMs são sanadas por uma frota heterogênea de PSVs, responsável pelo transporte de todas as cargas. Para que seja possível o abastecimento dos PSVs, é necessário que um porto faça parte da cadeia. O porto apresenta uma limitação física no número de berços disponíveis para uso e um conjunto de horários restritos, chamado de cronogramas, nos quais os PSVs podem atracar no porto e realizar suas operações.

Devido ao grande número de UMs e às grandes distâncias até a costa, se faz necessário que mais de uma UM seja atendida em cada viagem. Em decorrência desse fato, as UMs são separadas em clusters, sendo a clusterização e o roteamento do sistema prédefinidos.

A frota de PSVs é a maior responsável pelas emissões de poluentes, como o dióxido de carbono, óxido de nitrogênio, óxido de enxofre e partículas, de todo o setor upstream de petróleo (NORLUND et al., 2015). Estas emissões são provenientes do consumo de combustível e das condições dos equipamentos.

O principal fator que impacta no consumo de combustível de uma embarcação é a velocidade de navegação, outros fatores que também têm influência nesse consumo são as condições meteorológicas, o tipo de embarcação e o peso de navegação. Para este trabalho é considerado apenas o impacto da velocidade no consumo de diesel.

$\mathrm{O}$ consumo de combustível e a sua relação com a velocidade de navegação podem

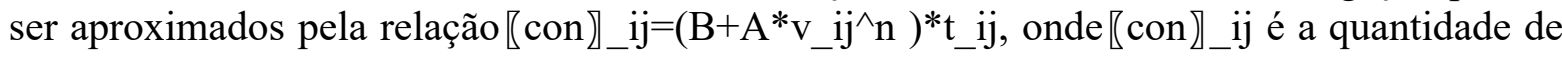
combustível consumido para fazer o trajeto de $\mathrm{i}$ até $\mathrm{j}, \mathrm{v}$ - $\mathrm{jj}$ é a velocidade de navegação do ponto $\mathrm{i}$ ao ponto j, t_ij é o tempo do decorrido para ir do ponto i até o ponto j e B, A e n parâmetros que dependem do tipo de embarcação e das condições de operação, sendo sempre $\mathrm{B} \geq 0, \mathrm{~A}>0$ e $\mathrm{n} \geq 3$, segundo (PSARAFTIS e KONTOVAS 2013). Psaraftis e Kontovas (2013) ainda dizem que a maioria dos artigos na literatura assumem uma função cúbica, por ser uma boa aproximação para o consumo de PSVs nas velocidades operacionais 
comumente utilizadas, com $\mathrm{B}=0$ e $\mathrm{n}=3$ e não dependência do peso da carga. É utilizado o valor de $\mathrm{A}=0,75$, como meio de validar o consumo do modelo com os valores reais de consumo de combustível da frota analisada.

A importância cada vez maior de buscar reduções nas emissões de poluentes nas operações, ao mesmo tempo em que não haja prejuízo no nível do serviço prestado e levando em conta o bem-estar da tripulação das embarcações, leva a uma necessidade de conciliar esses três aspectos muito distintos e de difícil comparação. Para que seja possível a avaliação conjunta destes fatores, é necessária a utilização de ferramentas multicritérios, que sejam capazes de avaliar todos os indicadores com suas devidas importâncias.

Assim, são abordadas três diferentes estratégias para determinar a velocidade de navegação dos PSVs:

- Estratégia 1: a velocidade utilizada segue o padrão observado nas operações da Petrobras nas Bacias de Campos e Espírito Santo;

- Estratégia 2: a velocidade de navegação é reduzida em relação a Estratégia 1; e

- Estratégia 3: a velocidade de navegação é aumentada em relação a Estratégia 1.

Ao se definir um grupo de estratégias que se quer comparar, é necessário definir os meios que permitiram que estas comparações sejam realizadas. São utilizados dois métodos considerados adequados para realizar a comparação: o primeiro é a utilização de um simulador de eventos discretos, conhecido como APOLO de Vianna (2019), que permite a implementação de detalhes pormenorizados da operação, embutindo por meios de distribuições de probabilidade as incertezas inerentes a operação e que são conhecidas; e o segundo é a utilização do modelo ELECTRE III, que é responsável pela análise multicritério da operação.

Para possibilitar a comparação entre as diferentes estratégias de determinação da velocidade de embarcação, é necessária a utilização de indicadores que retratem os três aspectos aqui abordados e que devem ser avaliados pelo método multicritério:

Fator econômico: é representado pelo nível de serviço de entrega de carga geral, que consiste na proporção de pedidos entregues no prazo pelo total de pedidos entregues (no prazo mais atrasados). Este indicador é de suma importância econômica, pois atrasos nas entregas dos pedidos podem acarretar paralização das operações nas unidades marítimas, gerando grandes prejuízos para as empresas envolvidas;

Fator sustentável: é caracterizado pelo total de combustível consumido pela frota de PSVs, já que o mesmo tem ligação direta com a quantidade de poluentes emitidos; e

Fator de bem-estar: é representado pelo tempo total de viagem, ou seja, quanto tempo é gasto do momento que o PSV sai do fundeio para o porto até o momento que ele volta para o fundeio. Este período pode ser considerado como sendo de trabalho ininterrupto da tripulação do PSV, longos períodos de trabalho isolado podem apresentar malefícios para a tripulação.

\subsection{MODELAGEM DA OPERAÇÃO OFFSHORE}

A operação offshore é composta por uma grande quantidade de etapas, que podem ser vistas em pormenores em Vianna (2019). Porém, apenas dois destes aspectos da modelagem são de maior relevância para este trabalho e devem ser detalhados:

- Velocidade do PSV: Para o cálculo da velocidade de navegação em cada uma das etapas da viagem, é levado em conta uma distância crítica, ou seja, quando a viagem tiver uma distância maior que a crítica, é sorteada a velocidade de 
navegação a partir de uma determinada distribuição e, por outro lado, quando a distância for menor que a crítica é sorteada uma velocidade de navegação a partir de outra distribuição. Para ambas as velocidades há um limite superior e inferior, que se forem alcançados a velocidade é sorteada novamente;

- Consumo de combustível do PSV: Para cada uma das atividades que o PSV realizou, há um consumo de combustível relacionado ao tempo que ele realizou essa tarefa. Os consumos são divididos entre: fundeio; berço; atravessando o canal; navegando, único caso que o consumo não é fixo, sendo dependente da velocidade de navegação; e na UM, que engloba o tempo que ele espera na fila para atracar, o tempo de espera para o começo das operações e o tempo de operação na UM. Não se considera que o PSV esteja consumindo combustível enquanto estiver em inoperância.

\subsection{MODELO MULTICRITÉRIO}

Na literatura da decisão, um sistema é muitas vezes definido em termos das interações existentes entre os elementos que o formam. Segundo Tomlinson e Kiss (1984), um processo de apoio à tomada de decisão é um sistema aberto. Para adaptar-se a complexidade do mundo real, é necessária uma simplificação a um modelo quantificável. Assim, a teoria da decisão é a convergência entre as escolhas, as alternativas e as respostas, referentes a diversos contextos de análise. A análise de problemas multicritério para o processo decisório passa pela consideração dos modelos referentes às qualidades dos operadores/atores diretamente envolvidos. Essas qualidades se referem a transdisciplinaridade e a multidisciplinaridade.

Dentre os modelos de apoio a tomada de decisão destacam-se os ELECTRE. Os modelos ELECTRE (Elimination et Choix Traduisant la Réalité) são um agrupado de métodos pertencentes à área de Auxílio Multicritério à Tomada de Decisão (AMD). O modelo ELECTRE III é desenvolvido por Roy (1978). Este modelo incorpora a natureza imprecisa e incerta na ordenação de alternativas de melhor para pior, utilizando os limiares de indiferença e preferência. Este modelo é o mais indicado para integrar diferentes variáveis linguísticas dentro de diferentes alternativas e, ainda, classificar e selecionar a melhor dentre tais alternativas.

\subsubsection{Breve Descrição do Método ELECTRE III}

Em relação um conjunto $\boldsymbol{A}=\left\{a_{1}, a_{2}, \ldots, a_{m}\right\}$ de alternativas avaliadas em relação a uma família de critérios $\boldsymbol{F}=\left\{g_{1}, \ldots, g_{n}\right\}$, o método ELECTRE III de Roy (1985) se propõe a estabelecer uma ordenação de preferência de alternativas. Neste método, a atribuição relativa dos pesos (importância) dos critérios é considerada de fundamental importância na modelagem. O método é estruturado em duas etapas: para cada par de alternativas (a, b), estas relações expressam a intensidade com que se pode afirmar que a subordinada b (intensidade com que a é ao menos tão boa quanto b). Se $g_{j}(a)$ é o valor de desempenho da alternativa a à luz do critério j, então calculam-se:

- Índices de concordância parcial $c_{j}(a, b)$ : que expressam a intensidade com que se pode afirmar que, sob o critério j, a é ao menos tão boa quanto b. $c_{j}(a, b)$ pode assumir os valores da equação (1): 
$\left.c_{j}(a, b)==\in\right] 0,1\left[\right.$,se $g_{j}(a)+q_{j}\left[g_{j}(a)\right]<g_{j}(b)<g_{j}(a)+p_{j}\left[g_{j}(a)\right]$

(1)

$$
=1 \text {, se } g_{j}(a)+q_{j}\left[g_{j}(a)\right] \geq g_{j}(b)
$$

-Índices de concordância global $c(a, b)$ : que expressam a concordância que existe em se afirmar que $a$ subordinada $b$, à luz de todos os critérios. $k_{j}$ é o peso associado a cada critério, obtendo assim a equação (2):

$$
c(a, b)=\sum_{j=1}^{m}\left(k_{j} \cdot c_{j}(a, b)\right) / \sum_{j=1}^{m} k_{j}
$$

A partir dos índices $\mathrm{Gc}(\mathrm{a}, \mathrm{b})$, constrói-se uma sucessão de relações de subordinação. Utilizam-se planos de corte $\lambda_{k} \in[0,1]$ e um limite de discriminação $s(\lambda)=\alpha^{\prime}+\beta^{\prime} \lambda_{k}$. A fim de impedir a utilização inadequada da função $s(\lambda)$ os valores recomendados para os parâmetros $\alpha^{\prime}$ e $\beta$ ' são, respectivamente, 0 e 0 (o decisor experiente pode alterá-los, se desejar ).

Se $D_{0}$ o conjunto das melhores alternativas de $\boldsymbol{A}$. No primeiro passo $k=0$ :

$\lambda_{0}=\max G_{c}(a, b)$ e $\lambda_{1}=\max G_{c}(a, b)$

(3)

$a, b$ e $D_{0} a \neq b \quad\left\{G_{c}(a, b)<\lambda_{0}-S\left(\lambda_{0}\right)\right\}$

(4)

Obtém-se a primeira relação $a S_{D_{0}}^{\lambda_{1}}$ b se e apenas:

$$
\mathrm{G}_{\mathrm{c}}(\mathrm{a}, \mathrm{b})>\lambda_{1} \text { e } \mathrm{G}_{\mathrm{c}}(\mathrm{a}, \mathrm{b})>\mathrm{G}_{\mathrm{c}}(\mathrm{b}, \mathrm{a})+\alpha^{\prime}+\beta^{\prime} \mathrm{G}_{\mathrm{c}}(\mathrm{a}, \mathrm{b})
$$

\section{RESULTADOS}

A partir da matriz de subordinação, calculam-se: a eficácia de a ( $\mathrm{n}^{\circ}$ de alternativas que a subordinada), a fraqueza de a ( $\mathrm{n}^{\circ}$ de alternativas que subordinam a) e a qualificação de a (a eficácia de a - a fraqueza de a). Após a qualificação, selecionam-se a(s) melhor(es) seleção(ões) descendente ou a(s) pior(es) seleção(ões) ascendente, das quais extraem-se as alternativas restantes.

Todos os testes realizados nessa etapa são executados em uma máquina equipada com Windows Server ${ }^{\circledR}$ Intel ${ }^{\circledR}$ Xeon ${ }^{\circledR}$ CPU E5-2620 v4 @ 2.10GHz com 2 processadores e 256 GB de memória RAM. É utilizado o Python 3.6 para a utilização do simulador APOLO. Para obtenção dos resultados utilizando o ELECTRE III, utiliza-se o software RStudio versão 3.5.3.

Para os resultados iniciais são simuladas no APOLO as três estratégias apresentadas previamente, com a Estratégia 2 apresentando valores de velocidade $20 \%$ menores e a Estratégia 3 com valores 20\% maiores, comparadas a Estratégia 1.

O cenário utilizado foi retirado de Vianna (2019), sendo o cenário base das operações e dos testes feitos, sendo utilizada a Estratégia 3B de alocação com um total de 32 PSVs na frota. Para todas as estratégias de seleção de velocidade, foram realizadas 30 replicações com duração de 180 dias de simulação com 90 dias de aquecimento. Neste cenário o espaço reservado no PSV para o primeiro backload é de 10 unidades, com um total de 90 UMs 
divididas em 23 clusters, o porto possui 6 berços com um total de 46 cronogramas disponíveis por semana.

A Tabela 1 apresenta os resultados dos indicadores para as três estratégias de velocidade adotadas.

Tabela 1 - Resultados das três estratégias.

\begin{tabular}{|c|c|c|c|}
\hline Estratégia & Nível de serviço & $\begin{array}{c}\text { Consumo de diesel } \\
\text { (m3) }\end{array}$ & $\begin{array}{c}\text { Tampo de viagem } \\
\text { (dias) }\end{array}$ \\
\hline 1 & $86,35 \%$ & 33673 & 4,304 \\
\hline 2 & $75,00 \%$ & 24247 & 4,629 \\
\hline 3 & $88,20 \%$ & 39725 & 4,175 \\
\hline
\end{tabular}

Fonte: Os autores

Para a construção dos cenários considera-se os pesos dos critérios econômico, ambiental e bem-estar da tripulação. As estratégias são as apresentadas em Vianna (2019). A Tabela 2 apresenta os resultados da matriz de concordância dos cenários um, dois, três e quatro. No primeiro cenário é dada igualdade de importância aos critérios econômico, ambiental e bem-estar da tripulação. No segundo cenário é dada maior importância ao critério econômico. No terceiro cenário é dada maior importância ao critério ambiental. Já no quarto cenário, é dada maior importância ao critério bem-estar.

Para a matriz de concordância não é possível dizer a preferências dentre as estratégias propostas, pois os índices de concordância são distintos. Neste caso, os valores muito diferentes entre as duas matrizes revelam forte influência dos índices de discordância, enfraquecendo a noção de concordância da preferência entre as alternativas.

Tabela 2 - Matriz de concordância dos cenários 1, 2, 3 e 4.

\begin{tabular}{|c|c|c|c|}
\hline \multicolumn{4}{|c|}{ CENÁRIO 1} \\
\hline & ECONÔMICO & AMBIENTAL & BEM-ESTAR \\
\hline ESTRATÉGIA 1 & 1,0 & 0,6 & 0,3 \\
\hline ESTRATÉGIA 2 & 0,3 & 1,0 & 0,3 \\
\hline ESTRATÉGIA 3 & 0,6 & 0,6 & 1,0 \\
\hline \multicolumn{4}{|c|}{ CENÁRIO 2} \\
\hline & ECONÔMICO & AMBIENTAL & BEM-ESTAR \\
\hline ESTRATÉGIA 1 & 1,0 & 0,6 & 0,3 \\
\hline ESTRATÉGIA 2 & 0,3 & 1,0 & 0,3 \\
\hline ESTRATÉGIA 3 & 0,6 & 0,6 & 1,0 \\
\hline \multicolumn{4}{|c|}{ CENÁRIO 3} \\
\hline & ECONÔMICO & AMBIENTAL & BEM-ESTAR \\
\hline ESTRATÉGIA 1 & 1,0 & 0,6 & 0,4 \\
\hline ESTRATÉGIA 2 & 0,4 & 1,0 & 0,4 \\
\hline ESTRATÉGIA 3 & 0,6 & 0,6 & 1,0 \\
\hline \multicolumn{4}{|c|}{ CENÁRIO 4} \\
\hline & ECONÔMICO & AMBIENTAL & BEM-ESTAR \\
\hline ESTRATÉGIA 1 & 1.0 & 0,5 & 0,5 \\
\hline ESTRATÉGIA 2 & 0,5 & 1,0 & 0,5 \\
\hline ESTRATÉGIA 3 & 0,5 & 0,5 & 1 \\
\hline
\end{tabular}

Fonte: Os autores

A Tabela 3 apresenta os resultados da matriz de preferência dos cenários um, dois, três e quatro. No cenário 1, a estratégia vencedora é a estratégia 3 tendo preferência de utilizando os critérios ambiental e bem-estar. No cenário 2, a estratégia vencedora é a estratégia 3. Já para os cenários 3 e 4, não há como determinar qual estratégia será utilizada pois há indiferença de utilização das três estratégias para os cenários propostos. 
A seleção de uma estratégia de movimentação de embarcações tipo PSV envolve muitos critérios, o que normalmente faz com que o decisor tenha dificuldades neste processo. Deste modo, a presente pesquisa utilizou o Método ELECTRE III para estabelecer um critério de escolha à luz de um conjunto de critérios (econômico, ambiental e bem-estar). São apresentados, os índices de concordância e as relações de preferência entre as estratégias de navegação, informações que podem contribuir para a tomada de decisão e que realçam a viabilidade do emprego do método para análise de tal problema.

\section{CONCLUSÃO}

O presente trabalho contextualiza o crescimento do setor de petróleo no mundo e no Brasil, indicando que as atividades em ambiente offshore possuem uma gama de dificuldades nas operações logísticas neste meio produtivo. Dada a grande quantidade de variáveis e de dados utilizados, nas atividades que integram toda a cadeia operacional na área offshore podem ser utilizar utilizados modelos de pesquisa operacional a fim de encontrar soluções otimizadas. Para estes casos se faz necessária o uso de uma análise multicritério, podendo então serem considerados fatores econômicos, fatores sustentáveis dentre outros.

Tabela 3 - Matriz de Preferência dos cenários 1,2, 3 e 4.

\begin{tabular}{|l|l|l|l|}
\hline \multicolumn{5}{|c|}{ CENÁRIO 1 } \\
\hline & ESTRATÉGIA 1 & ESTRATÉGIA 2 & ESTRATÉGIA 3 \\
\hline ESTRATÉGIA 1 & I & P & P \\
\hline ESTRATÉGIA 2 & NP & I & P \\
\hline ESTRATÉGIA 3 & NP & NP & I \\
\hline \multicolumn{5}{|c|}{ CENÁRIO 2 } \\
\hline & ESTRATÉGIA 1 & ESTRATÉGIA 2 & ESTRATÉGIA 3 \\
\hline ESTRATÉGIA 1 & I & P & P \\
\hline ESTRATÉGIA 2 & NP & I & P \\
\hline ESTRATÉGIA 3 & NP & NP & I \\
\hline \multicolumn{5}{|c|}{ CENÁRIO 3 } & ESTRATÉGIA 3 \\
\hline & ESTRATÉGIA 1 & ESTRATÉGIA 2 & I \\
\hline ESTRATÉGIA 1 & I & I & I \\
\hline ESTRATÉGIA 2 & I & I & I \\
\hline ESTRATÉGIA 3 & I & I & ESTRATÉGIA 3 \\
\hline \multicolumn{7}{|c|}{ CENÁRIO 4 } & I \\
\hline & ESTRATÉGIA 1 & ESTRATÉGIA 2 & I \\
\hline ESTRATÉGIA 1 & I & I & I \\
\hline ESTRATÉGIA 2 & I & I & I \\
\hline ESTRATÉGIA 3 & I & I
\end{tabular}

Fonte: Os autores

Assim, o trabalho busca contribuir no ordenamento de três estratégias de seleção da velocidade de navegação da frota de PSVs (Plataform Supply Vessels) que são barcos que suprem as demandas das Unidades Marítimas (UMs), baseando-se em critérios econômicos, ambientais e de bem-estar da tripulação, analisados por um modelo multicritério. Para alcançar este objetivo, é utilizada uma ferramenta de apoio à decisão, denominada APOLO, que combina um simulador de eventos discretos com uma heurística de alocação dos PSVs às cargas e às unidades marítimas. É selecionado o modelo ELECTRE III para a realização da análise multicritério, depois são definidos os indicadores relevantes para o problema. Como resultado, obtém-se a terceira estratégia como vencedora, apresentando os índices de concordância e as relações de preferência entre as estratégias de navegação. Deste modo, as informações obtidas pelo modelo podem contribuir para a tomada de decisão e que realçam a viabilidade do emprego do método para análise de tal problema. O método multicritério se mostra relevante e aplicável a processos de tomada de decisão que envolvam variáveis de caráter subjetivo e quantitativo 


\section{REFERÊNCIAS}

[1] AGUIAR, R. A. Estudo do Impacto da Ampliação de Berço e de Área de Pátio no Aumento da Capacidade Operacional de uma Base de Apoio Offshore. Dissertação (Mestrado em Engenharia Civil) - Programa de Pós-Graduação em Engenharia Civil. Universidade Federal do Espírito Santo, Vitória, 2013.

[2] ALMEIDA, M. R. de. Algoritmos Genéticos Aplicados a Programação de Embarcações de Apoio às Operações “Offshore”. Anais do XLI Simpósio Brasileiro de Pesquisa Operacional (SBPO), Bahia, 2009.

[3] ALMEIDA, P. P. de. Aplicação do método AHP - Processo Analítico Hierárquico à seleção de helicópteros para apoio logístico à exploração e produção de petróleo "offshore". Dissertação (mestrado) - Universidade Federal de Santa Catarina, Centro Tecnológico. Programa de Pós-Graduação em Engenharia de Produção. 2002.

[4] AGÊNCIA NACIONAL DO PETRÓLEO - ANP. Anuário Estatístico 2017. 2017. Rio de Janeiro. Disponível em: <http://www.anp.gov.br/wwwanp/images/publicacoes/ anuario-estatistico/2017/ anuário \{_\} 2017.pdf.>. Acessado em 15 de abr de 2019.

[5] ARES, G. Logística de apoio offshore - integração e sincronização da cadeia de atendimento às unidades marítimas. 2013. Disponível em: <http://www.ilos.com.br/ web/logistica-de-apoio-offshore-integracao-e-sincronizacao-da-cadeia-deatendimento-as-unidades-maritimas/.eferência>. Acessado em 15 de abr de 2019.

[6] ARPINI, B. P.; ROSA, R. DE A. Planejamento da logística de suprimento de plataformas Offshore por meio de um modelo matemático 2L-CVRP com frota heterogênea e equilíbrio náutico. Transportes, v. 23, n.4, p. 67-78. 2015.

[7] BRAGA, A. A. Uma análise de um sistema de transporte de passageiros para plataformas offshore. Monografia apresentada na Graduação em Engenharia Naval e Oceânica da Escola Politécnica, Universidade Federal do Rio de Janeiro, Rio de Janeiro, 2017.

[8] COSTA, P. F. F da. Otimização da logística de operação de navios aliviadores de petróleo usando programação matemática. Dissertação de mestrado do Departamento de Engenharia Industrial, Pontifícia Universidade Católica do Rio de Janeiro, 2015.

[9] DI LUCCIO, F.B.; DORES, P.B.DAS. O mercado de apoio offshore: panorama e perspectivas. BNDES Setorial, Rio de Janeiro, n.43, p. 295-323, março, 2016.

[10] ESKANDARI, H.; MAHMOODI, E. A simulation-based multi-Objective optimization study of the fleet sizing problem in the offshore industry. Maritime Economics and Logistics, v.18, p.436-457, 2016.

[11] KISIALIOU, Y.; GRIBKOVSKAIA, I.; LAPORTE, G. Robust supply vessel routing and scheduling. Transportation Research Part C: Emerging Technologies, v.90, p.366-378, 2018.

[12] MARTINS, F.G.; COELHO L.S. Aplicação do método de análise hierárquica do processo para o planejamento de ordens de manutenção em dutovias. Revista GRPOS, Gestão da Produção, Operações e Sistemas, v. 7, n. 1, p. 65-80, 2012.

[13] MOREIRA, P. C. M. Análise de aumento da eficiência do sistema marítimo offshore de transporte de carga por meio de simulação probabilística. Dissertação 
de Mestrado no Programa de Engenharia de Transportes, Universidade Federal do Rio de Janeiro, 2017.

[14] NORLUND, E.K.; GRIBKOVSKAIA, I.; LAPORTE, G. Supply vessel planning under cost, environment and robustness considerations. Omega, v. 57, p. 271-281, 2015.

[15] NORLUND, E.K.; GRIBKOVSKAIA, I. Environmental performance of speed optimization strategies in offshore supply vessel planning under weather uncertainty. Transportation Research Part D: Transport and Environment, v. 57, p.10-22, 2017.

[16] NORSTAD, I.; GRIBKOVSKAIA, V.; JOHNSEN, T.; LINDSTAD, H.E.; UTHAUG, E. Simulation-Based Evaluation of Upstream Logistics System Concepts for Offshore Operations in Remote Areas. ASME 2017 36th International Conference on Ocean, Offshore and Arctic Engineering, p. V008T07A003, 2017.

[17] PSARAFTIS, H.N.; KONTOVAS, C.A. Speed models for energy-efficient maritime transportation: a taxonomy and survey. Transportation Research Part C: Emerging Technologies, v. 26, p. 331-351, 2013.

[18] PINTO, A.P.; ROSA, R. DA A.; ROSA, A.L. DA S. Modelo matemático para planejamento tático das rotas dos barcos para suprimento das plataformas offshore de exploração de petróleo. Logística, Modelagem em Transportes e Logística, v.31, p. 630-641, 2015.

[19] SILVA, V. A.; LIANG, D. A.; YUBA, D. G. T.; ROSSETTO, R.; KOVALSKI, S. Logistics Planning for Critical Cargos Related to Hydrogen Sulfide Removal Processes in Pre-Salt Basin Fields. Offshore Technology Conference, Rio de Janeiro, Brazil, Oct., 2015.

[20] ROY, B. ELECTRE III: Algorithme de Classement Base Sur Une Représentation Floue des Préférences en Présence des Critères Multiples. Cahiers du CERO, v. 20, n.1, p. 3-24, 1978.

[21] ROY, B. Méthodologie multicritère d'aide à la décision. Paris: Economica. 1985.

[22] TOMLINSON, R.; KISS, I. Rethinking the Process of Operational Research and Systems Analysis. United Kingdom: Pergamon Press, 1984.

[23] VIANNA, I. G. P. Análise da alocação de embarcações de apoio offshore com o uso de heurísticas e simulação de eventos discretos. Dissertação de Mestrado do Programa de Engenharia de Produção, Universidade Federal do Rio de Janeiro, 2019.

[24] WATROBSKI, J.; JANKOWSKI, J.; ZIEMBA, P.; KARCZMARCZYK, A.; ZIOLO, M. Generalised framework for multi-criteria method selection. Omega, v.86, p. 107124, 2019.

As Referências Bibliográficas devem seguir as normas da ABNT/NBR 6023. 